\title{
When learning Italian as a Second Language, tourism and technology go hand in hand
}

\section{Cristiana Cervini, Anna Zingaro}

Department of Interpretation and Translation (DIT), University of Bologna Italy.

\begin{abstract}
This paper aims to describe the development of CALL-ER, an application for mobile devices, produced within the CALL-ER project (Context-Aware Language Learning in Emilia Romagna). An ever-increasing availability of applications for language learning that meet the different learning needs of users, as well as the ubiquitous wireless communication, led applications for mobile devices to become gradually more context-aware. This means that language is acquired by users through the direct experience with the local context where they are. An example in this regard is represented by the CALLER mobile application, that supports mobility students through the incidental learning of Italian language and culture in the city of Forli. We will begin this contribution with an outline of the theoretical underpinnings that supported the project and a presentation of the project itself. We will then present the first stage of the project, during which the application was developed before its first testing. At this point, an overall description of the application will be given. A special attention will be paid throughout this paper both to how language learning has been conceived through experiential tourism and to the multimodality of the contents.
\end{abstract}

Keywords: Context-aware language learning; Italian L2; MALL; multimodality. 


\section{New contexts for language learning}

The potential of technology to promote the adoption of specific approaches to language learning has grown over the years and an example of this is the ever-increasing availability of applications for language learning. This paper will describe how technology, namely a geolocalized app for mobile devices, could be useful to promote experiential learning and context-aware mobile learning. The case study specifically regards the learning of Italian as a second language in the urban and suburban areas of Forli-Cesena. In accordance with the latest innovations in the field of techno-pedagogical innovation (Ferguson et al., 2019), the app will have specific functions to foster meaningful, mobile, context-aware learning of Italian as a second language, as well as to facilitate interaction between groups of users (communities of interest, communities of practice).

The app is primarily targeted at a very heterogeneous audience of "tourist-learners": students coming to Forlì for a short period of study or international students fully enrolled at the University of Bologna (Forlì Campus). These participants present very different linguistic profiles and levels of knowledge of Italian. Moreover, the app topics are designed to be of interest to a second kind of "tourist-learners", that also include native speakers of Italian, who might be interested in a new dimension of interaction with their surroundings. The aspect which will hopefully be shared by all the participants is the desire to get in touch with new places of the town, as well as the to share the experiences with other people through different forms of sharing and/or online peer tutoring.

Palalas (2016, V) specifies that in MALL, learner mobility is combined with: "access to people and resources (both these residing locally on the device and those on the Web), to digital tools (including built-in device capabilities, native and web-based apps), as well as supports and scaffolds mediated by mobile devices [...]". In mobile learning, the concept of context-awareness is defined as the activity of collecting information about one's surroundings to provide a measure of what is happening at a specific time around a user and a device. Mobile devices prove to be an excellent tool for performing activities and delivering content that is particularly relevant in each environment, as by their nature they lend themselves to being available in different contexts and can act as a support for the learner in case of need (Naismith et al., 2004, p. 14).

Incidental learning has been defined in general terms as "a byproduct of some other activity, such as task accomplishment, interpersonal interaction, sensing the organizational culture, trial-and-error experimentation, or even formal learning" (Marsick and Watkins [1990] 2015, p. 12). This definition of incidental learning seems to fit the CALL-ER app1 features. Indeed,

\footnotetext{
${ }^{1}$ At present, the app has the provisional name CALL-ER, that identifies the project. The development of the final name under which the app will be launched will take place in the later stages of its design.
} 
the main aim of its users will not be that of improving their Italian language skills. Rather, they will hopefully improve Italian in an incidental way, through new oral and written input linked to the places where they are spending a period of their lives. They will be enabled to create a bridge with other people who share the same cultural and social interests, with the aim of building a community of interest. Even if the project is at its initial stages, the main technological and educational features of the app have already been defined. The present research is mainly focused on the description of the challenges linked to the app contents' design: how can we produce good quality and interesting contents with the aims of promoting both touristic places and language incidental acquisition? Given that the download of the app will be free, such a potential heterogeneous and wide target group makes the app design even more challenging; in fact written and audiovisual contents will be available for elementary, advanced and Italian mothertongue participants.

Authoring and designing processes have been facilitated by an important previous experience gained in the Ilocalapp project.

\section{Previous experiences}

The design of CALL-ER benefits from the cooperation of a few previous projects in the field of technology, multilingualism and language education2. More precisely, the first research network was created in 2010 and it involved a highly international and interdisciplinary network in which social, linguistic, and cultural sciences interact with technology and engineering (Ceccherelli \& Valva, 2016). This collaboration has enabled the realization of several European projects aimed at designing technological platforms and devices for the dissemination and learning of less widely spoken languages among which we mention the ILOCALAPP project, in which the UniON! app was developed (Ceccherelli et al., 2016). The project ILOCALAPP3, developed in the period 2015-2018 within the Erasmus+ Programme, Key 2-Strategic Partnerships in Higher Education, is the birthplace of the idea for the CALL-ER app. The evaluation of the Erasmus + National Agency states "the APP, the main IO of the project, has a huge potential for transfer both at national and transnational level".

The UniON! app, with open-source code, is available for Android and Apple on Google and Apple store, with more than 500 downloads on googlestore only for the Italian version geolocalised in Bologna (Valva et al., 2018). It is a highly innovative solution for incidental

\footnotetext{
${ }^{2}$ We want to thank all the participants to the CALL-ER project, namely: Federico Garcea, Silvia Mirri, Daniele Negretti and Catia Prandi for the technological parts, Silvia Bernardini, Patrick Leech and Alessia Mariotti for the support in the content development.

${ }^{3}$ Cfr. Ilocalapp - http://www.ilocalapp.eu/ (Incidentally Learning Other Cultures and Languages through an App).
} 
and context-aware learning of less widespread languages, among which, precisely, Italian in Bologna (Cervini, 2018).

CALL-ER takes up the principles at the basis of the design of the Union app (Cervini et al., 2016), orienting the redesign on the communicative, cultural and tourist needs of Forlì, one of the city of the UNIBO multicampus, aiming at a greater interactivity among users and at the valorization of places out of the classical tourist circuits.

\section{The CALL-ER app}

CALL-ER is a free mobile application for language and culture incidental learning. In order to offer the possibility to get acquainted with the local settings, it is equipped with geolocalisation and context-awareness functionalities, that provide the users with language, culture and practical information related to the city of Forlì and its surrounding areas. Most of the structure and the functionalities of the application is inspired by the ILOCALAPP project. The code used for its design is open source, so it can be reused also for the design of other similar apps.

The contents include both texts and multimedia (images, audio, videos), alongside with links to external resources. They are organized in six main categories, that branch out into several subcategories, containing texts and their related multimedia: 1) Places: culture (museums, historical places), nature (parks, sports, well-being), leisure and entertainment (theaters, music, cinema); 2) Itineraries: Rationalist route, Medieval route, Outside the classic routes, naturalistic route; 3) Events: festivals, fairs etc., divided by season; 4) University life: Studying at Unibo, libraries and meeting places; 5) Food \& drink: Romagna's recipes, traditional restaurants and bars, students' favourite places; 6) Services: transportation, walking and cycling; health, housing, money and post office.

As can be seen, the content structure moves from very general topics, such as, Food \& drink and so on, to more specific socio-cultural features that are strictly related to the local reality (e.g.: Food and drink > Romagna's recipes etc.). The choice of the general topics is based on retracing the habits of international students both in their daily-life as university students and also as young adults living a new life abroad. For this reason, both institutional places (university, libraries, public offices, etc.) and entertainment ones (cinemas, museums, pubs, etc.) are included.

In addition to these six categories, there are another two functions. The Word of the Day shows everyday a new word (or phrase), including the main vocabulary covered by the app and even some words in the local dialect that are more likely to be heard in everyday conversations, and an instant messaging system, which allows users to chat with each other and create a community. 
In order to make sure that the contents of the Call-ER app would meet the requirements of its potential end-users, a participatory approach was adopted. In the early stage of the project it consisted in collecting the opinions and expectations of the potential end-users, i.e., mobility students in Forlì and some tourist organisations and associations involved in promoting the province of Forli-Cesena, that may use it to make their services better known to the public.

Foreign students were subjected to an anonymous online questionnaire, whereas tourist organisations were given the choice between an anonymous questionnaire and a telephone interview4. The questionnaire for mobility students collected information about the cultural areas of interest, their linguistic difficulties encountered during their stay in Forlì, their knowledge of the territory and background information about the general usage of apps and mobile devices. Moreover, a specific section inquired into their possible appreciation of an application that combined language learning with the discovery of the city of Forlì and an open question for any additional reflections or advice.

To give an overall review of the answers, all the 21 participating students agreed on the need for a greater integration of international students into the Italian-speaking student community. The most desired features for an app that combines knowledge of the territory and learning Italian resulted to be the following ones: geo-localized itineraries with audio guides, photos and images, information in multiple languages and, above all, the opportunity to interact with a community in to turn to for help and support, as well as to make friends and practice Italian. Moreover, the answers also highlighted the need to focus not only on lexicon and phraseology related to daily life and tourism, but also to those situations in which the non-Italian-speakers interfaces with Languages for Specific Purposes, such as bureaucracy, health, rental agreements and so on.

The questionnaire for tourist organisations was mostly focused on how to promote the area of Forlì and their view of an application that combined both promoting the area and learning Italian. The answers obtained by 4 organisations showed the need to improve the promotion of the area, the current lack of adequate plans, events and advertising and a possible appreciation of an application combining tourism and language learning.

After having established the topic area of the main categories on the basis of the results of the questionnaire, the second stage consisted in re-examining the contents of the UniOn! app, in order to see if some of them could fit for the purposes of the CALL-ER app. This was the case, for example of the University Life category, in which a general overview on the University of Bologna, its services and some culture-bound concepts (like 'ricevimento' and

\footnotetext{
${ }^{4}$ We want to thank Enrica Romano, whose Master's Degree thesis was focused on developing the questionnairs, interviewing the contact persons for the local tourist organisations and on analysing the collected data.
} 
'buchetta') are also adequate to Forlì. Therefore, the texts were kept in their approximately original form and only a few edits were necessary to adapt the contents to the local context of Forli and to update them with data related to the academic year 2020-2021. The same will be done for the contents related to public services, transports, etc.

Contrary to this, the contents related to places and itineraries have been written from scratch, since they are strictly related to Forlì and its surroundings. While choosing the places of the territory to be promoted through the app, the attention was focused on unusual places that do not fall within the traditional canons of tourist destinations but which are however peculiar to the area. For this reason, it was also taken into consideration the activity of two cultural associations, namely ATRIUM and Spazi Indecisi, with the former having the purpose to investigate and manage the "dissonant" architectural heritage of the totalitarian regimes of the 20th century, and the latter aiming at transforming urban abandoned places into a field of investigation and research for artists, architects, urban planners and so on. This phase of documentation, supported by the consultation of Lonely Planet and Touring Club guides, as well as tourist guides written by local experts, was integrated by the discussion within the research team on the results of the research.

On this basis, it was taken the decision to plan several routes (rationalist / medieval/ naturalistic routes and a special one, named 'Outside the classic routes'). Moreover, a notification system alerts the users when they are near places and activities of interest, showing different options (e.g., cultural places, typical restaurants, entertainment etc.). In this regard, the design of the app would be beneficial for all parties involved: the cultural activities of local authorities and associations would receive greater visibility, reaching the public of international students, with the latter having more opportunities to get to know their host city, its surroundings and traditions.

\section{Creation of app contents}

The creation of contents for incidental language learning through experiential tourism implies focusing on several aspects: the learning goals to be achieved, the promotion of the area, as well as the framework of the electronic devices on which the application, that runs on both Android and iOS devices, will be working.

As for the learning goals, one of the most challenging issues was the choice to produce contents that are addressed to the whole community, independently from the competence in the target language (i.e., to beginners as well as to more advanced students). This deeply influenced the writing strategies and led to the development of a series of guiding principles.

Firstly, each text will be marked with coloured icons to distinguish easy texts (green icon) from those of medium (yellow) or high difficulty (red). The icons may be perceived as 
reading suggestions: it is up to the users to choose texts suitable for their language skills, or to challenge themselves with higher difficulty levels. This strategy is aimed at avoiding the users' demotivation or boredom.

Secondly, techniques for writing highly comprehensible texts have been applied. For example, the texts classified as easy or medium have short and coordinated sentences. Moreover, the lexicon was carefully chosen and composed mainly of high-frequency words. Less frequent words, such as idiomatic expressions or terms belonging to a specific domain (e.g., architecture, bureaucracy etc.) are usually followed by reformulations and/or synonyms, to enhance understanding and in some cases they are hyperlinked to the Word of the Day function. Moreover, the small screen of electronic devices was a key factor in planning short-length contents, ranging from 100 to 300 words.

Finally, a combination of different semiotic resources, such as moving /still image, speech and writing has been exploited to promote the development of integrated skills, to favour different cognitive styles and to create a more involving experience for users. At this point, a significant innovation compared the UniON! app is the integration of written texts with audiofiles containing additional information or short interviews to students and citizens. Moreover, some descriptions are given in oral texts only.

In order to stimulate curiosity, the menu allows for full accessibility of contents at the main category level. The user has the opportunity to start indifferently from any category and to take a look and choose whether to continue or switch to other categories and subcategories. However, in order to promote active participation into the learning process, users may access the first 1-2 texts (or just some paragraphs of a text) of a subcategory, then the subsequent contents would be bound to unlocking activities based on the concept of gaming, such as answering a question or performing an action, like uploading a picture etc. Alongside with gamification, there are also another two strategies to involve users, that represent a significant innovation compared the UniON! app. Firstly, the above mentioned instant messaging system (see $\S 3$ ) will allow users to chat with each other, thus creating a community in which they can share experiences on the places they visited. Secondly, a periodic launch of thematic challenges (e.g., the most beautiful photo etc.) will hopefully stimulate the interest in visiting the area and accessing an increasing number of contents in the app.

\section{Conclusion}

In this paper we have described the development of the CALL-ER application. The results collected through the first round of questionnaires show that an app for the incidental learning of Italian through the promotion of the area of Forlì may interest both students and tourist organisations. A previous similar experience was successfully conducted with the app 
UniON! designed for context-aware and incidental language learning of international students in Bologna in the period 2015-2018. Since that time, MALL and context-aware apps are increasingly widespread. The innovative sides of the new CALL-ER app lies above all in these three aspects: i) the chance to be part of an online community of students, where sharing comments, pictures, ideas on itineraries; ii) the representation in oral and written texts of a non-standard variety of the Italian language (the Romagnolo dialect), that students can daily listen in the streets or in the city's shops, but hardly found in dictionaries; iii) the introduction of some gamification features in order to increase motivation and to regulate the different stages of learning; iv) a more prominent focus on oral authentic inputs, either recording testimonies from international students and Forlì's citizens or linking on the app contents touristic ans cultural audio-videos.

From this point of view, using the app will be a win-win strategy both for language learning and tourism. It will hopefully enhance the creation of a network between university, local authorities and regional associations, for the promotion of cultural and educational activities outside the classroom.

Current limitations of the app include the fact that its design is still in its early stages. Therefore, it is not yet possible to describe in detail neither its different functionalities, nor users' activities in the app (e.g. outcome of gaming activities) and their opinions. Subequent stages of the questionnaire will be necessary to collect the users'opinions and to integrate any suggested changes in the the app, before its final release. Another interesting research future perspective concerns the observation of how informal contexts of learning (through the app) could mix with more formal contexts, such as at schools or during teachers-led activities. For example, will students feel like using the app for self-study too?

Further investigation will be needed in order to better understand the effects of mobile incidental learning through an app in language and/or culture learning. This will be the object of future developments within the project teams.

\section{References}

Ceccherelli A., Valva A. (2016), Fostering multilingualism and student mobility: the case of E-LOCAL for all. Interdisciplinarità e apprendimento linguistico nei nuovi contesti formativi. L'apprendente di lingue tra tradizione e innovazione, 21-39. doi: 10.6092/unibo/amsacta/5069.

Ceccherelli, A., Cervini, C., Magni, E., Mirri, S., Roccetti, M., Salomoni, P., Valva, A. (2016). The ILOCALAPP Project: a Smart Approach to Language and Culture Acquisition. The Future of Education Conference Proceedings, 270-275.

Cervini, C. (2018). Esperienze linguistico-culturali a Bologna: apprendere l'italiano L2 con UniON. Esperienze di e-learning per l'italiano: metodi, strumenti, contesti d'uso, Bologna: Bononia University Press - BUP. 81 - 95. 
Cervini, C., Solovova, O., Jakkula, A., Ruta, K. (2016). Mobile assisted language learning of less commonly taught languages: learning in an incidental and situated way through an app. CALL communities and culture - Short papers from EUROCALL 2016, Voillans, Research-publishing.net, $81-86$.

Ferguson, R., Coughlan, T., Egelandsdal, K., Gaved, M., Herodotou, C., Hillaire, G., Jones, D., Jowers, I., Kukulska-Hulme, A., McAndrew, P., Misiejuk, K., Ness, I. J., Rienties, B., Scanlon, E., Sharples, M., Wasson, B., Weller, M. and Whitelock, D. (2019). Innovating Pedagogy 2019: Open University Innovation Report 7.

Marsick, V.J., e K.E. Watkins. (1990) 2015. Informal and Incidental learning in the workplace. Abingdon (Inghilterra), New York: Routledge.

Naismith, L., M. Sharples, G. Vavoula, e P. Lonsdale. (2004). Literature Review in Mobile Technologies and Learning. Futurelab Series - Report 11. hal-00190143, 1-44.

Palalas, A. (2016). Introduction to the Handbook. The International Handbook of MobileAssisted Language Learning, I-XV. Pechino: China Central Radio \& TV University Press Co., Ltd.

Valva, A., Mirri, S., Salomoni, P. (2018). User centered design applied to an app for incidental learning of languages and cultures. IMSCI 2018 - 12th International MultiConference on Society, Cybernetics and Informatics, Proceedings, International Institute of Informatics and Systemics, IIIS, 2, $55-60$. 